\title{
Polar magnetic field geometry in the solar cycle and the relationship between spherical harmonics
}

\author{
B. P. Filippov, Yu. V. Platov \\ and D. V. Klepikov
}

Institute of Terrestrial Magnetism, Ionosphere and Radio Wave Propagation, Russian Academy of Sciences, Troitsk Moscow Region, 142190, Russia email: bfilip@izmiran.ru

\begin{abstract}
Geometry of the polar magnetic field is changing during the activity cycle. Tangents to polar ray structures are usually crossed near some point below the surface. This point can be named as a "magnetic focus". The distance $q$ between the focus and the center of the solar disk changes from the maximum value about $0.7 R_{\odot}$ at solar minimum activity to the minimum value about $0.5 R_{\odot}$ at solar maximum. This behavior has not been understood till now. Really, all tangents to field lines near the pole of the axially symmetric potential magnetic field described by zonal spherical harmonics cross at the same point. However, the greater is the number of a harmonic, the closer to the surface is the crossing point. As far as at activity maximum high harmonics dominate, the behavior of the focuses seems to be in contradiction with the results of the harmonic analysis. We have found that the focus depth changes could be explained by complicated relationship between the harmonics of "new" and "old" cycle near the instant of the polar magnetic field reversal.
\end{abstract}

Tangents to the polar ray structures are usually crossed near some point below the surface of the Sun. This point can be named as a "magnetic focus". The distance $q$ between the focus and the center of the solar disk changes from the maximum value about $0.7 R_{\odot}$ at solar minimum activity to the minimum value about $0.5 R_{\odot}$ at solar maximum (Nesmyanovich 1962; Gulyaev 1998; Makarov 1998; Makarov, Tavastsherna, \& Callebaut 1998; Koutchmy and Bocchialini 1998).

The distance $q$ from the center of the Sun to the focus of the field lines described by $n$-th spherical harmonic is defined by the relationship (Ermakov, Obridko \& Shelting 1995)

$$
q^{(n)}=R_{\odot} \frac{n}{n+2} .
$$

It is evident that the value of $q$ increases along with the increasing of harmonic's number $n$ approaching to the unity. As far as at activity maximum higher harmonics dominate (Bravo, Stewart \& Blanco-Cano 1998), the behaviour of the focuses seems to be in contradiction with the results of the harmonic analysis.

The deep location of the focuses near activity maximum could be explained by complicated relationship between the harmonics of a "new" cycle and an "old" cycle during the process of polarity reversal. The expression for $q$ for the sum of two harmonics is given by (Filippov, Platov \& Klepikov 2004)

$$
q^{(n+m)}=R_{\odot} \frac{n}{n+2}\left(1+\frac{\frac{m(n+2)}{n(m+2)}-1}{\frac{(n+1)(n+2)}{C(m+1)(m+2)}+1}\right),
$$

where $\mathrm{C}$ is the ratio of harmonic coefficients. When $\mathrm{C}$ is negative and lays within the 
interval $-\frac{n(n+1)}{m(m+1)}<C<0$, the value of $q$ for the sum of two harmonics is less then $q$ for each harmonic. Negative sign of $\mathrm{C}$ means that the magnetic field in polar regions described by the two harmonics has the opposite polarity, or they may be attributed to the "new" cycle and the "old" one.

Since the phase difference between the low order harmonics and higher harmonics is about of a half of a period of an 11-year cycle or a quarter of a period of a 22-year cycle, we can approximate the most general behavior of the global magnetic field by only two harmonics with the coefficients changing as

$$
\begin{gathered}
g_{3}(t)=\cos \omega t, \\
g_{7}(t)=A \cos \left(\omega t \pm \frac{\pi}{2}\right),
\end{gathered}
$$

where $\omega$ corresponds to the 22-year period of the Hale magnetic cycle and $A$ is the ratio between the amplitude values of the coefficients $g$. At activity minimum the value of $q$ is about $0.7 R_{\odot}$, then, $q$ increases or decreases depending on the sign of the phase difference. Low values of $q$ are achieved before or after the polarity reversal.

The polarity reversal means the pass of a neutral point of the global magnetic field through the solar surface at the pole. Magnetic field lines near the neutral point have the shape of hyperbolas changing steeply in space. The influence of this structure on the polar magnetic field geometry seems to be the key factor for the magnetic focus lowering at solar maximum.

\section{Acknowledgements}

This work was supported in part by RFBR under grant number 03-02-16093.

\section{References}

Bravo, S., Stewart, G. A. \& Blanco-Cano, X. 1998 Solar Phys. 179, 223-235.

Ermakov, F.A., Obridko, V.N. \& Shelting B.D. 1991 Astronomy Reports 39, 672-674.

Filippov, B. P., Platov Yu. V. \& Klepikov D. V. 2004 Astronomy Reports 48, 781-786.

Gulyaev, R. A. 1998 In Solar Jets and Coronal Plumes. ESA SP-421, pp. 277-279.

Koutchmy, S. \& Bocchialini, K. 1998 In Solar Jets and Coronal Plumes. ESA SP-421, pp. 51-62.

Makarov, V. I. 1998 In Proc. 18th NSO Workshop, (ed. K. S. Balasubramaniam, J. Harvey \& D. Rabin). ASP Conference Series, vol. 140, pp. 83-87.

Makarov, V. I., Tavastsherna, K. S. \& Callebaut, D. K. 1998 In Novyj tsikl aktivnosti Solntsa: nabludatelnyj i teoreticheskii aspekty, pp. 111-114, GAO, St.Petersburg.

Nesmyanovich, A. T. 1962 Astron. Zh. 39, 996-1002. 